\title{
Diagnostic Value of the Morphofunctional Characteristics of Platelets in the Prevention for Complications of Chronic Obstructive Pulmonary Disease
}

\author{
Alyavi AL, Sadykova GA*, Rahimova DA and Muminov DK \\ Republican Specialized Scientific-Practical Medical Center of Therapy and Medical \\ Rehabilitation and Tashkent Pediatric Medical Institute, Tashkent, Uzbekistan \\ *Corresponding Author: Sadykova GA, Republican Specialized Scientific-Practical \\ Medical Center of Therapy and Medical Rehabilitation and Tashkent Pediatric \\ Medical Institute, Tashkent, Uzbekistan.
}

Received: January 23, 2021

Published: March 11, 2021

(C) All rights are reserved by Sadykova GA., et al.

\begin{abstract}
The results of studies of qualitative morphofunctional characteristics of thrombocytes in patients with COPD with I-II-III severity arepresented.The technique of a thrombocytopogramfor diagnostic and prognostic purpose is offered.It wasestablished that the main and decisive moment of the onset of thrombosis of any blood vessel in the body is the initial formation of a platelet thrombosis. The expediency of integrating heparin electrophoresis into complex base therapy of COPD isestablished and it improves the functional activity of thrombocytes and has a thrombolytic effect.
\end{abstract}

Keywords: Chronic Obstructive Pulmonary Disease; Thrombocyte; Electrophoresis; Heparin

\section{Introduction}

More than half of lethal cases after therapeutic diseases occurred because of disaster in the heart`s, lung`s, kidney`s and brain`s vessels (E.I. Chazov 2005). The modern theory of blood coagulation system includes the following factors: coagulation system (phases); a system of anticoagulants, a system of anticoagulant inhibitors, platelet and plasma factors. It is generally recognized that the platelet, as the smallest cell in the body, is the conductor and participant in the large process of blood coagulation. The platelet itself is a round or oval cell with pink cytoplasm, 2 to 5 microns in diameter. It has a dark purple granulomere in its cytoplasm, called the hyalomera.

Platelets normally flow in the most extreme zone of blood stream, sliding along the inner surface of the vascular endothelium, due to the simple cyclin, which is produced by the cells of the vascular endothelium and therefore does not adhere to the vas- cular wall. But, if the vascular endothelium is damaged, that happens with various damage to the vascular endothelium, platelets instantly acquire the properties of adhesion to the collagen layer of the vascular wall. It happens in something like this: enzyme located on the surface of the platelet (glycosyl transferase) is the first to adhere to the collagen layer of the vascular wall, and platelet adhesion occurs. This is also facilitated by the von Willy Brandt factor, adenosine diphosphoric acid and plasma factor IV.

Platelet phospholipase is activated as a result of platelet adhesion, and it converts platelet arachidonic acid into prostaglandin $\mathrm{G}^{2}$, under the action of which platelet opening occurs.

The second platelet emptying also takes place with the release of solid platelet granules (prostaglandin $\mathrm{G}^{2}$ and other factors, especially thromboxane A2). Thromboxane A2 starts the $3^{\text {rd }}$ factor - platelet agglomeration, in other words platelet adhesion to each 
other. This is the beginning of the formation of a platelet thrombus. At the same time, a third platelet factor, platelet thrombokinase or thromboplastin comes out of the platelet and forms active thromboplastin when it is combined with plasma thromboplastin-3 with the participation of tissue thromboplastin and erythrocyte thromboplastin. Under the action of active thromboplastin, the second phase of the blood coagulation - the conversion of prothrombin into thrombin process occurs.

It was found that at the later stages of COPD the formed microscopic platelet aggregates cause microthrombosis and consequently disrupt pulmonary and coronary blood flow. Thus, patients with severe course of COPD are at high risk of platelet quality change, since one of the major causes of differently locates thrombosis are platelets [1-4]. According to various authors, the frequency of arterial hypertension $(\mathrm{AH})$ in patients with COPD fluctuates in a rather wide range from 6.8 to $76.3 \%$, averaging at $34.3 \%$. It is assumed that hypoxia in patients with COPD can increase blood pressure $[7,8]$. Based on the above mentioned, antithrombotic therapy can affect inflammatory and proliferative processes, and therefore it is a necessary link in the treatment of diseases associated with hypercoagulation syndrome [2].

In recent years, the presence of more than 60 active substances in the platelet has been firmly established, about 14 of them are platelet factors involved in the process of hemostasis. When studying platelets, besides the total number of platelets, their quality is of great importance as well. So, nowadays, there is a great need for an accelerated study of the morphological and functional characteristics of platelets as they characterize the state of the coagulation and anti-coagulation system of blood in patients.

The adhesion of platelets to the elements of the vascular wall is the initial stage in the process of hemostasis and arterial thrombosis and it determines the significance of informative value of this indicator in laboratory diagnostics and evaluating the effectiveness of physiotherapeutic treatment of pulmonary patients.

Existing methods of assessing platelet adhesion are complex and used mainly for research purposes.

Functional and morphological characteristics of peripheral blood platelets serves as one of the earliest and available methods predicting platelet intravascular thrombosis $[1,4,6]$.

\section{Aim of the Study}

To study the dynamics of platelets quality characteristics in terms of their adhesion and aggregation in patients with COPD, depending on the severity and in the dynamics of complex physiotherapeutic treatment with the inclusion of heparin electrophoresis.

\section{Materials and Methods}

We studied 55 patients with COPD (I, II, III degrees of severity according to the European Respiratory Society classification and the Global Initiative for Chronic Obstructive Lung Diseases (GOLD)) in remission. There were 31 men and 24 women aged 43 to 75 years $(45,0 \pm 5,0)$ with currency of pulmonary disease for about $12.3 \pm 4.3$ year. The patients were randomized (by gender, age and nosology) into 2 groups. In the main group 35 patients received complex physiotherapy with electrophoresis heparin (10000 units in $30 \mathrm{ml}$ of distilled water, bilaterally on the thorax, active electrode was connected with the anode at 3 to $12 \mathrm{~mA}$, current density no more than $0.03 \mathrm{~mA} / \mathrm{cm}^{2}$ and duration 15 minutes, daily, 8 - 10 procedures per course) of the apparatus "Potok". In the control group (20 patients), a similar treatment was carried out without the inclusion of heparin electrophoresis. Patients of both groups received together with basic therapy exercise therapy, massage, hydroaeroionization with iodine, cleansing and therapeutic enemas, circular shower and dosed phototherapy.

All patients underwent a study of external respiration function on the device "Medicor" by "Elektromedia" with the measurement of $\mathrm{VC}, \mathrm{FEV}_{1}$, maximum volume velocities at the level of small, medium and large bronchi (MOS75, MOS50, MOS25), electrocardiogram, a general blood test with a total platelet count, sputum analysis, coagulogram, platelet aggregation on the analyzer "AP 2110" ("SOLAR", Belarus) using adenosine diphosphate (ADP). The functional activity of platelets was assessed (degree and rate of aggregation, degree of disaggregation, time of onset of aggregation).

In this work, a simple, accessible, informative method was developed to establish adhesion and agglomeration of cytomorphological, functional, qualitative characteristics of platelets in a peripheral blood smear [1].

Platelets in the human body are produced by the disintegration of megakaryocyte cytoplasm giant cells, located in the bone marrow. It is possible to judge the activity of the bone marrow by 
Diagnostic Value of the Morphofunctional Characteristics of Platelets in the Prevention for Complications of Chronic Obstructive Pulmonary Disease

megakaryocytes. It has been conditionally established that out of 100 megakaryocytes 50 are normally in an active state.

Initially, red azurophilic granularity appears in the cytoplasm of megakaryocytes. There is a border compaction of the megakaryocyte cytoplasm around it. The platelet is formed by lacing and exits with the blood flow into a peripheral blood vessel. Normally, the number of platelets ranges from 180 to 320 thousand in $1 \mathrm{~mm}^{3}$. Quantitative determination of platelets in peripheral blood is carried out according to the method of prof. Fonio in all laboratories in the following sequence. A thin blood smear taken from a finger is fixed for 5 minutes in methanol. Then, after drying in air, it is poured with Giemsa-Romanovsky paint (from 20 to 45 minutes depending on the freshness and quality of the paint) or Bexter paint for 3-5 minutes, previously diluted with distilled water in a ratio of 1:10. Then the blood smear is dried and looks along the meander line under the immersion lens and the number of platelets per 1000 erythrocytes is counted in 5 - 7 places. The resulting figure is multiplied by the total number of erythrocytes minus three zeros.

In smears of peripheral blood, stained by Giemsa - Romanovsky, we studied 100 platelets on the meandering line, their size, number of singles, small-, medium- and large-agglomerated platelets and the presence spinous ( $t$ e. amoeba) bulging (in\%) of on their surface $[4,5]$.

\section{Results and Discussion}

Taking into account the change in the platelets cytomorphology, a comparative analysis of the qualitative characteristics of platelets by the number of adhered, agglomerated platelets was carried out respectively with their reaction of release from the contents and depending on potential platelet thrombi, on blood pressure in- dicators, aggregometric indicators, fibrinogen content, volumetric and speed indicators of external respiration function in every patient. When calculating the indicators of the agglomeration ability of platelets in peripheral blood in percent, we found that normal platelets in $80-85.0 \%$ of cases are single. The appearance of paired (that is, adhesion of two platelets to each other) of platelets indicated adhesion, and they were called small aggregates (normally they were found from 10 to $14.0 \%$ ). Clumping from 3 to 8 - 10 platelets were considered medium agglomerates (normally, their frequency ranged from 1 to $9.0 \%$ ). Clumping from 11 to 25 platelets or more were called large agglomerates (they are not normally formed) [1,3].

Based on research, we obtained facts indicating that the main and decisive moment in the occurrence of thrombosis of any blood vessel in the body is the initial formation of a platelet thrombus. With the progression of disease amount of single platelets decreased and referring percentage of small, medium with the large agglomerates increased in blood smears, and it is essential in the formation of platelet thrombi [6].

Platelet grams in COPD patients with the first degree of severity before and after treatment in both groups were in the normal range in $93 \%$ of patients (Table 1 ). In accordance with the reaction of the platelets release from their contents, the qualitative characteristic of platelets changes. Large platelets are thrombocytes that are not emptied from their contents and are considered young and functionally active. Small platelets are considered passive and exhaust. It was noted that the degree of platelet activity was more pronounced in severe COPD with pulmonary hypertension (Table 1).

\begin{tabular}{|l|c|c|c|c|c|c|c|c|c|}
\hline \multicolumn{1}{|c|}{ Nosology } & $\begin{array}{c}\text { Common platelets } \\
\text { (thousand in 1 } \mathbf{~ m m}^{\mathbf{3}} \text { ) }\end{array}$ & Single & Small & Medium & Large & Big & Small & Active & $\begin{array}{c}\text { Not } \\
\text { active }\end{array}$ \\
\hline COPD - I stage (14) & $270.0 \pm 3.4$ & 82 & 13 & 5 & - & 45 & 55 & 45 & 55 \\
\hline COPD - II stage (12) & $279.0 \pm 2.8$ & 75 & 16 & 9 & 4 & 45 & 55 & 45 & 55 \\
\hline COPD - III stage (9) & $269.4 \pm 3.0$ & 70 & 17 & 13 & 6 & 63 & 37 & 60 & 40 \\
\hline Control - (20) & $281.0 \pm 2.4$ & 76 & 15 & 9 & 3 & 51 & 49 & 50 & 50 \\
\hline Healthy - (10) & $264.0 \pm 2.5$ & 85 & 10 & 5 & - & 50 & 50 & 50 & 50 \\
\hline
\end{tabular}

Table 1: Quantitative-qualitative indicators of platelets in peripheral blood in patients with chronic obstructive pulmonary disease, depending on the severity (in \%).

Citation: Sadykova GA., et al. "Diagnostic Value of the Morphofunctional Characteristics of Platelets in the Prevention for Complications of Chronic Obstructive Pulmonary Disease". Acta Scientific Medical Sciences 5.4 (2021): 86-90. 
In patients with II degree COPD after the course of heparin electrophoresis, increased (in 23\% of patients) indicators of agglomerated platelets decreased to normal in $21 \%$ versus control (11\%). In $26 \%$ of patients with COPD III with initially high rates of platelet agglomeration, after treatment was revealed a tendency to disaggregation (compared to the control group, 9\%) in the study group. With the advent on platelet surface spinous processes, adhesion becomes agglomeration of I and II degree with the release of thromboxane A2 and other substances, whereby the large platelets become small or exhaust. This process correlates with the clinical treatment of the disease and with the rapid characterization of aggregometric studies, increasing the process of disaggregation on the aggregometer and does not correlate with the content of fibrinogen. The identity of the established results of platelet alteration during microscopic and aggregometric studies. In the examined patients with COPD of the main group, after the course of treatment, compared with the baseline data and the control group, positive dynamics of the functional state of platelets and the rate indicators of the function of external respiration (FEV1, MOS75, MOS50, MOS25) of the Tiffno test (FEV1/VC) $(\mathrm{P}<0,05)$ was found. Treatment is individualized according to gender, age, season and the presence of concomitant diseases. It was found that in the occurrence of a primary platelet thrombus, the functional characteristics of the platelet play a decisive role, their adhesiveness and aggregation ability, which depends on many internal factors.

A direct correlation between the severity of COPD and the maximum degree of ADP-induced aggregation was found. Initially, in patients with COPD stage III. a significant $(\mathrm{P}<0.001)$ increase in platelet aggregation function was revealed, whereas in patients with milder grade I-II COPD, these indicators were the lowest, but did not reach normal values, as in the group of healthy individuals. In the dynamics of treatment in the main group, there is a decrease in the rate and degree of aggregation, an increase in the time of the onset of aggregation and an increase in platelet disaggregation $(\mathrm{P}$ $<0.01$ ), although the control group did not reach the level of reliability ( $\mathrm{P}>0.05)$.

Aggregation and agglomeration of platelets is accompanied by their secretory activity according to the merocrine type. With a significant thrombosis on a large scale, they develop a picture of a pulmonary infarction, myocardium, a stroke of one organ or another and sudden death from blockage of many blood vessels by millions of the above-described microscopic thrombi that can occur.
A direct correlation has been established between the degree of COPD activity with the maximum degree of ADP-induced aggregation and the cytological characteristics of platelets in peripheral blood $[2,5]$.

In the dynamics of treatment with heparin electrophoresis, a decrease in the rate and degree of aggregation, an increase in the time of onset of aggregation, an increase in disaggregation and a decrease in agglomerated platelets were revealed.

\section{Conclusion}

Thus, the proposed method of platelet gram is economically effective (it is used without expensive equipment for aggregometric and biochemical studies) and can be used for diagnostic and prognostic purposes in all clinical laboratories of the Ministry of Health of the Republic of Uzbekistan by pulmonologists, therapists, physiotherapists, surgeons, obstetricians - gynecologists, etc. Complex application of electrophoresis heparin improves the functional activity of platelets and has a thrombolytic effect. This substantiates the advisability of using heparin electrophoresis in combination with basic therapy at the stages of rehabilitation of patients with COPD.

\section{Bibliography}

1. Nadzhimitdinov ST and Sadykova GA. "A new method of informational assessment of the morphofunctional characteristics of platelets". Information letter, Tashkent (2007): 5.

2. Alyavi AL., et al. "Acupuncture hirudotherapy in the complex treatment of patients with chronic obstructive pulmonary disease". Method Recom Tashkent (2008): 12.

3. Wilson JM and Ferguson III JJ. "Platelet - endothelial interactions in atherothrombotic disease: therapeutic implications". Clinical Cardiology 22 (2014): 687-698.

4. S Lindemann., et al. "Activated platelets mediate signaling by regulated interleukin 1b synthesis". Journal of Cell Biology 154 (2011): 485-490.

5. Alyavi AL., et al. "A program for predicting the formation of platelet thrombosis in blood vessels". Tashkent city No. DGU 01124 (2006). 
6. Alyavi AL., et al. "Algorithm for informational assessment of individual indicators of intravascular homeostasis". DGU Computer Program (2017).

7. Chuchalin AG. "Chronic obstructive pulmonary disease problems of diagnosis and treatment". Ter Archive (2010): 33-59.

8. Chronic bronchitis and obstructive pulmonary disease". Edition. Cocos A. H.SPb (2012).

\section{Assets from publication with us}

- Prompt Acknowledgement after receiving the article

- Thorough Double blinded peer review

- Rapid Publication

- Issue of Publication Certificate

- High visibility of your Published work

Website: www.actascientific.com/

Submit Article: www.actascientific.com/submission.php

Email us: editor@actascientific.com

Contact us: +919182824667 